\title{
Determination of optimal wavelet denoising parameters for red edge feature extraction from hyperspectral data
}

\begin{abstract}
A study of wavelet denoising on hyperspectral reflectance data, specifically the red edge position (REP) and its first derivative is presented in this paper. A synthetic data set was created using a sigmoid to simulate the red edge feature for this study. The sigmoid is injected with Gaussian white noise to simulate noisy reflectance data from handheld spectroradiometers. The use of synthetic data enables better quantification and statistical study of the effects of wavelet denoising on the features of hyperspectral data, specifically the REP. The simulation study will help to identify the most suitable wavelet parameters for denoising and represents the applicability of the wavelet-based denoising procedure in hyperspectral sensing for vegetation. The suitability of the thresholding rules and mother wavelets used in wavelet denoising is evaluated by comparing the denoised sigmoid function with the clean sigmoid, in terms of the shift in the inflection point meant to represent the REP, and also the overall change in the denoised signal compared with the clean one. The VisuShrink soft threshold was used with rescaling based on the noise estimate, in conjunction with wavelets of the Daubechies, Symlet and Coiflet families. It was found that for the VisuShrink threshold with single level noise estimate rescaling, the Daubechies 9 and Symlet 8 wavelets produced the least distortion in the location of sigmoid inflection point and the overall curve. The selected mother wavelets were used to denoise oil palm reflectance data to enable determination of the red edge position by locating the peak of the first derivative.
\end{abstract}

Keyword: Signal processing; Spectroscopy; Wavelet transforms; Wavelets 